\title{
Five decades of eukaryotic transcription
}

\author{
The biochemical identification of three nuclear RNA polymerases fundamentally altered our understanding of \\ gene regulation.
}

F ifty years ago, our understanding of transcription was informed by pioneering studies of bacteriophage and bacterial RNA polymerases, which revealed that a polymerase engages single or bipartite promoter DNA elements either directly or via a polymerase-associated $\sigma$ subunit to initiate gene-specific RNA synthesis. Coordinate gene expression was largely achieved either by the linear arrangement of genes within operons or by the association of bacterial $\sigma$ factors to direct the core RNA polymerase to sequence-specific promoter elements.

In this context, the biochemical purification and identification of three nuclear RNA polymerases was unprecedented. Each RNA polymerase had a dedicated function in the synthesis of the precursor of large ribosomal subunit rRNAs (Pol I), 5S rRNA and tRNAs required for protein synthesis (Pol III) and proteinencoding mRNAs (Pol II). The subsequent demonstration that, despite their subunit complexity, these enzymes were incapable of initiating RNA synthesis at their cognate promoters within DNA templates in vitro launched an entire field of investigation to identify their accessory transcription factors that has uncovered an ever-expanding complexity of regulatory mechanisms.

To celebrate the anniversary of this landmark discovery, we present a special Focus on 50 years of eukaryotic transcription, with commissioned historical Perspectives and a Review highlighting key advances in the biochemical elucidation and characterization of the nuclear RNA polymerases and the protein factors and DNA elements that control their activities. The authors of these historical pieces narrate the remarkable progress in our understanding of these topics from a personal perspective.

A Review by Robert ("Bob") Roeder provides a central narrative of the Focus, as it describes discoveries spanning a research career that has provided seminal contributions to the field over five decades (https://doi.org/10.1038/s41594-0190287-x). His initial 1969 discovery, as a graduate student, of three nuclear RNA polymerase activities from multiple eukaryotes, distinguished by their metal and DNA template preferences, and shown by Pierre Chambon to be differentially sensitive to the fungal toxin, $\alpha$-amanitin, marks both the dateline of our anniversary Focus and the beginning of a field of scientific investigation that has informed not only eukaryotic transcriptional regulation but also other DNA-dependent processes (Nature 224, 234-237 (1969); Fed. Proc. 28, 599 (1969); Science 170, 447-449 (1970); Biochem. Biophys. Res. Commun. 38, 165-171 (1970)).

As an independent investigator, Bob Roeder and his group went on to define the DNA-binding factors that direct specific initiation from Pol II and Pol III promoters in vitro, to identify the first gene-specific regulatory factor, TFIIIA, required for $5 \mathrm{~S}$ rDNA gene transcription (the first reported zinc-finger DNA-binding factor), and to describe the ordered assembly of general transcription factors at promoters to create a pre-initiation complex that directs gene-specific transcription initiation by Pol II or Pol III.

The unanticipated complexity of these initiation factors was augmented by the observed complexity of DNA elements and DNA-binding proteins that mediate gene- or cell-type-specific transcription by Pol II and led to the definition of activator and enhancer elements that coordinate expression of genes in distant locations within the genome via interactions with sequence-specific activator- or enhancer-binding factors. The subsequent identification of cofactors, such as Mediator, that bridge enhancer-bound regulatory factors and promoter-bound transcription factors, provided additional insights into gene- and cell-specific regulatory mechanisms that we now know contribute to three-dimensional genome architecture.

As the central role of chromatin in gene expression is now well established, it is difficult to imagine a time when its functional significance was questioned. A Perspective by Jim Kadonaga (https://doi. org/10.1038/s41594-019-0278-y) relates the initial resistance of the transcription field, which at the time was focused on purified, reconstituted systems using naked DNA templates, to the potential benefits of assessing RNA polymerase activity on chromatin-assembled DNA substrates. Although this approach was seen as 'throwing a wrench into the works' of a biochemically defined system, it ultimately proved that chromatin was a spoke in the wheel of the transcription cycle. Transcriptional activators were shown to counteract repressive chromatin structures through chromatin remodeling activities that favor 'open' chromatin configurations at promoters, a novel function at the time, and ultimately paved the way to show how posttranscriptional modifications of histones permit or preclude transcriptional activity.

Transcription elongation is facilitated by factors that modify or remodel chromatin or that directly target the Pol II elongation complex to regulate its activity. A Perspective by Joan and Ron Conaway (https://doi.org/10.1038/s41594-019-0283-1) describes the biochemical identification and characterization of the latter class of accessory factors, including TFIIS, the first described elongation factor that reactivates arrested Pol II complexes on DNA templates. The subsequent discovery of Elongin and ELL-factors that suppress Pol II pausing at sites where it is prone to arrest-led to the identification of the 'super elongation complex' (SEC) that includes the $\mathrm{p}$ - $\mathrm{TEFb}$ kinase that phosphorylates the Pol II CTD. Several genes encoding SEC subunits are translocation partners for the methyltransferease MLL in leukemia, establishing a link between misregulated Pol II elongation and leukemogenesis.

The foundational biochemical studies that defined key factors and cofactors that mediate eukaryotic transcriptional regulation have been augmented by technological advances that allowed their functions to be examined both at gene-specific loci and genome-wide within cells. A Perspective by John Lis (https://doi.org/10.1038/ s41594-019-0288-9) traces the contributions of emerging technologies to elucidating transcriptional mechanisms by targeted disruption of factor activities, tracking transcription factor interactions, nucleosomal configuration and RNA polymerase activity at base-pair resolution and monitoring enhancer-promoter interactions on a genomic scale. Combined with current super-resolution microscopy and cryo-EM visualization of transcription intermediates, we anticipate that the molecular interactions underlying transcriptional regulation in vivo will continue to be illuminated.

We hope that each piece conveys the excitement of the contemporaneous discoveries and reinforces their contributions to our understanding of the mechanisms underlying RNA polymerase function and how coordinate or differential gene expression is achieved in eukaryotic cells.

Published online: 26 August 2019 https://doi.org/10.1038/s41594-019-0303-1 